\title{
Control of the F-14 Aircraft Lateral-Directional Axis During Powered Approach
}

\author{
Gary J. Balas* \\ University of Minnesota, Minneapolis, Minnesota 55455 \\ Andrew K. Packard ${ }^{\dagger}$ \\ University of California, Berkeley, Berkeley, California 94720 \\ Joe Renfrow ${ }^{\ddagger}$ and Chris Mullaney \\ U.S. Naval Air Warfare Center, Patuxent River, Maryland 20670-5304 \\ and \\ Robert T. M'Closkey ${ }^{\S}$ \\ University of California, Los Angeles, Los Angeles, California 90095
}

\begin{abstract}
The design of linear controllers for the F-14 aircraft lateral-directional axis during powered approach using the structured singular-value $(\mu)$ framework is presented. Controllers are designed for an angle of attack of $10.5 \mathrm{deg}$ and an airspeed of $137 \mathrm{kn}$, the on-speed flight condition. Each controller is implemented in a simplified nonlinear simulation and the full-order Fortran nonlinear simulation of the F-14. Pilot-in-the-loop simulations are used to verify their performance in the crewed flight simulator at the U.S. Naval Air Warfare Center, Patuxent River, Maryland. The $\mu$ controllers out perform the current analog and newly developed digital lateral-directional powered approach flight control system in pilot-in-the-loop simulations.
\end{abstract}

\section{Nomenclature}

$C_{\delta_{\mathrm{sp}}} \quad=$ differential spoiler roll input coefficient

$\mathrm{F} 14_{\text {nom }}=$ linearized F-14 models at $\alpha$ of $10.5 \mathrm{deg}$

$G_{r} \quad=$ rudder actuator transfer function

$G_{\mathrm{sp}} \quad=$ spoiler actuator transfer function

$G_{\text {st }} \quad=$ stabilizer actuator transfer function

$K=$ controller

$P \quad=$ generalized interconnection structure

$p \quad=$ roll rate, $\mathrm{deg} / \mathrm{s}$

$r \quad=$ yaw rate, $\mathrm{deg} / \mathrm{s}$

$V_{\text {cal }} \quad=$ airspeed, $\mathrm{kn}$

$v \quad=$ lateral velocity, $\mathrm{ft} / \mathrm{s}$

$W_{\text {act }} \quad=$ actuator magnitude and rate weight

$W_{\text {in }} \quad=$ input multiplicative uncertainty weight

$W_{n} \quad=$ sensor noise weight

$W_{p} \quad$ = roll rate tracking weight

$W_{\beta} \quad=$ sideslip tracking weight

$W_{\dot{\beta}} \quad=$ sideslip rate response weight

$y_{\mathrm{ac}}=$ lateral acceleration, $g$

$\alpha \quad=$ angle of attack, deg

$\beta \quad=$ sideslip, deg

$\gamma \quad=$ flight-path angle, $\mathrm{deg}$

$\Delta_{G} \quad=$ perturbation

$\delta_{\mathrm{dsp}} \quad=$ differential spoiler deflection, deg

$\delta_{\text {dstab }}=$ differential stabilizer deflection, deg

$\delta_{\text {lstk }} \quad=$ lateral stick deflection, in.

$\delta_{\text {rped }} \quad=$ rudder pedal deflection, in.

$\delta_{\text {rud }} \quad=$ rudder deflection, deg

$\kappa=$ blending variable

$\mu \quad=$ structured singular value

$\phi \quad=$ bank angle, deg

$\omega_{\mathrm{GM}} \quad=$ gain margin frequency

$\omega_{\mathrm{PM}} \quad=$ phase margin frequency

Received May 1, 1997; revision received Feb. 25, 1998; accepted for publication April 19, 1998. Copyright (C) 1998 by the authors. Published by the American Institute of Aeronautics and Astronautics, Inc., with permission.

*Associate Professor, Department of Aerospace Engineering and Mechanics. E-mail: balas@aem.umn.edu. Member AIAA.

†Professor, Department of Mechanical Engineering.

† Staff Engineer, Aircraft Division.

${ }_{\text {S Assistant Professor, Department of Aerospace, Nuclear and Mechanical }}$ Engineering.

\section{Introduction}

$\mathbf{T}$ $\mathrm{HE}$ task of landing an aircraft on an aircraft carrier requires precise control because the tail hook must engage one of four arrestment wires spaced 40-ft apart on the deck. With an approach speed of $137 \mathrm{kn}, \mathrm{a}-3$-deg glide path, and under no ship motion conditions, the F-14 aircraft will nominally clear the ship by $11 \mathrm{ft}$ and touchdown $1 \mathrm{~s}$ later with an impact velocity of $13 \mathrm{ft} / \mathrm{s}$ (Ref. 1). The originalF-14 aircraft was equipped with an analog flight control system (AFCS) to improve handling qualities in the pitch and lateraldirectionalaxis during a powered approachlanding. The AFCS uses differential stabilizers in the feedback loop, feed-forward differential spoiler deflections to generate roll moments, and rudder deflections in the feedback loop to generate sideslip. Figure 1 shows the control surface locations on the F-14 aircraft.

Even with the AFCS, there is significant coupling in the lateraldirectionalaxis, which results in pilots having difficulty landing the F-14 aircraft on a carrier. In 1993 the U.S. Navy initiated a program to upgrade the powered approach flight controllaws to make landing on a carrier easier. ${ }^{2}$ This new digital flight control system (DFCS) had its first flight test in July 1995. Based on pilot comments, the DFCS is a significant improvement over the previous analog flight control laws for powered approach flight.

This paper presents a parallel effort to the lateral-directional DFCS design and the synthesis of controllers for the F-14 lateraldirectional axis during powered approach using the structured singular-value $\mu$ framework. ${ }^{3-9}$ Controllers are designed for an angle of attack $\alpha$ of $10.5 \mathrm{deg}$ and an airspeed of $137 \mathrm{kn}$, the on-speed flight condition. These controllers are implemented in a simplified nonlinear simulation and the full-order Fortran nonlinear simulation. Pilot-in-the-loop tests for each design were performed in the crewed flight simulator at the U.S. Naval Air Warfare Center, Patuxent River, Maryland.

The initial $\mu$ controllersperformed well when implemented on the SIMULINK nonlinear simulation of the F-14 aircraft, though not as well when implemented on the full-order, Fortran F-14 aircraft simulation. Specifically, the controllers exhibited too much sideslip $\beta$ during lateral stick inputs. This was not observed in the SIMULINK simulation due to the poor correlation between the SIMULINK simulation $\beta$ response and the Fortran simulation $\beta$ response. These differences are due to the SIMULINK simulation using the linearized equations of motion of the aircraft, whereas the Fortran simulation implements the fully coupled, nonlinear equations of motion. 

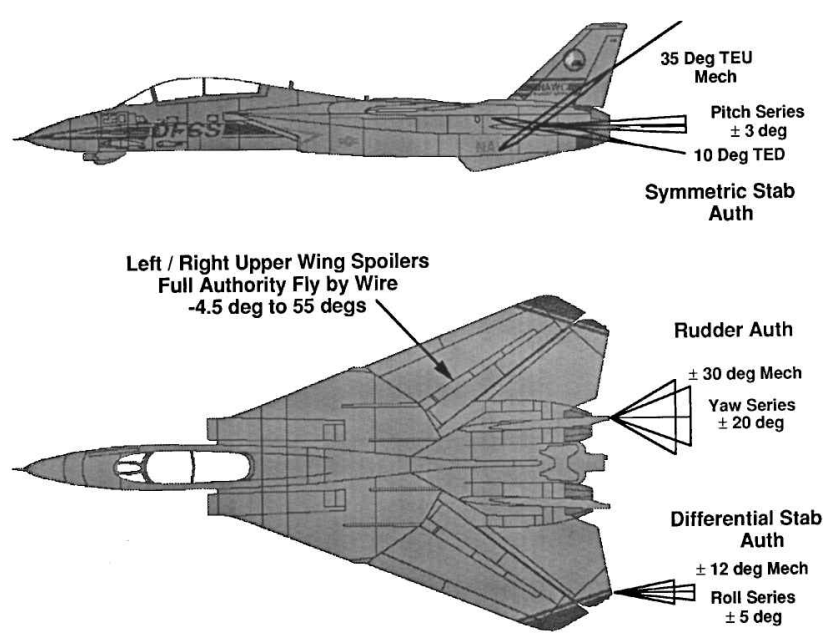

Fig. 1 F-14 aircraft actuator surfaces.

To reduce the sideslip response of the $\mu$ controllers, the sideslip tracking requirement was relaxed, and a penalty on the sideslip rate $\dot{\beta}$ was included in the $\mu$ control problem formulation. The results of the controllers designed with the $\dot{\beta}$ error term are presented in Sec. III. The performance of the controllers designed with the $\dot{\beta}$ error were significantly improved as compared with the initial $\mu$ controllers.

The two most noticeable differences between the $\mu$ controllers and the current DFCS are: the reduced coupling between the roll and sideslip and the $\mu$ designs have a linear response with lateral stick, whereas the current DFCS is designed to have a nonlinear response. That is, DFCS increases the lateral stick roll rate sensitivity when the lateral stick exceeds \pm 1.0 in.

Two linear $\mu$ multivariable point designs at 10.5-deg angle of attack, denoted as controller Kmod2q and Kmod2sx, and the AFCS and DFCS were implemented and tested in pilot-in-the-loopsimulations at the crewed flight simulator at the U.S. Naval Warfare Center in Patuxent River, Maryland. Lead F-14 test pilot Scott Kelly evaluated Kmod2q and Alan Poindexterevaluated Kmod2q and Kmod2sx designs, and their detailed comments are presented. The F-14 aircraft is initialized at 1000-ft altitude, 10.5-deg angle of attack, and $137 \mathrm{kn}$ : the on-speed condition, on a -3-deg glide path offset $200 \mathrm{ft}$ laterally from the touchdown centerline.

The pilot task is to fly the aircraft to an altitude of $200 \mathrm{ft}$, with a 200 -ft lateral offset, then maneuver the aircraft to land in the center of the runway. This simulates the real situation of breaking out of clouds close to landing on the carrier without being correctly aligned with the runway centerline. Both the pilots stated that both of the controllers achieved all of the performance objectives. Overall both controllers out performed the AFCS and DFCS for the on-speed powered approach condition in the F-14 crewed flight simulator.

The following is an outline of the paper. In Sec. II, the linearized model of the F-14 lateral-directional axis is presented along with actuator models and sensors models. This section describes how differences between the nonlinear model of the F-14 aircraft and the linearized model are taken into account in the control designs. Section III describes the selection of performance weighting functions for the two $\mu$ control designs: Kmod2q and Kmod2sx. The selection of weighting functions to achieve desired robustness and performance objectives in the control design are discussed in this section. The control problem is formulated as a model-matching problem in the $\mu$ framework and level- 1 handling quality models are selected. Section IV describes the $D-K$ iteration procedure used to design the $\mu$ controllers. In addition, the gain and phase margins, plots of the input and output sensitivity and complementary sensitivity, nonlinear time responses, and pilot comments during pilot-inthe-loop simulations of the $\mu$ controllers are presented. Section V contains a summary of results and conclusions of this work.

\section{F-14 Lateral-Directional Linear Designs}

We consider the design of a lateral-directionalaxis controller for the F-14 aircraft during powered approach to a carrier landing. A linearized F-14 model is derived at $\alpha=10.5 \mathrm{deg}$ and an airspeed of $137 \mathrm{kn}$. The objective is to design a linear robust, multivariable controller. It is assumed that the controller measures or senses the lateral stick input, rudder pedal input, aircraft roll rate, yaw rate, and lateral acceleration. The controller generates commands to the stabilizers, rudder, and spoilers.

The design of the lateral-directional axis controller for the F-14 during powered approach is posed as a robust performance control problem. The structured singular-value $\mu$ framework, specifically $D-K$ iteration, is used to synthesize controllers. ${ }^{3-9}$ The robustness objective is for the controller to be insensitive to multiplicative uncertainty at the plant input. The performance objectives are accounted for in the $\mu$ framework via minimizing weighted transfer function norms. ${ }^{9-11}$ Weighting functions serve two purposes in the $\mathcal{H}_{\infty}$ and $\mu$ framework: They allow the direct comparison of different performance objectives with the same norm and they allow for frequency information to be incorporated into the analysis. The F-14 performance weighting functions used in the $\mu$ control designs are discussed in detail in Sec. III. All of the weighted performance objectives are scaled to have an $\mathcal{H}_{\infty}$ norm less than 1 when they are achieved. $^{12}$

\section{A. Nominal and Uncertainty Models}

The pilot has the ability to command the lateral-directional response of the aircraft with the lateral stick $\delta_{\text {lstk }}$ (inches) and rudder pedals $\delta_{\text {rped }}$ (inches). The aircraft has three control inputs: differential stabilizer deflection $\delta_{\text {dstab }}$ (degrees), rudder deflection $\delta_{\text {rud }}$ (degrees), and differential spoiler deflection $\delta_{\mathrm{dsp}}$ (degrees); three measured outputs: roll rate $p$ (degrees per second), yaw rate $r$ (degrees per second), and lateral acceleration $y_{\mathrm{ac}}(g)$; and three calculated outputs: sideslip angle $\beta$, bank angle $\phi$ (degrees), and sideslip rate $\dot{\beta}$. Note that $\beta$ and $\dot{\beta}$ are not measured variables but are used as performance measures in the $\mu$ control designs. The lateraldirectional F-14 model, F14 nom, has four states: lateral velocity $v$ (feet per second), yaw rate, roll rate, and bank angle. These variables are related by the state-space equations

$$
\left[\begin{array}{c}
\dot{v} \\
\dot{r} \\
\dot{p} \\
\dot{\phi} \\
\hline \beta \\
\dot{\beta} \\
p \\
r \\
\phi \\
y_{\mathrm{ac}}
\end{array}\right]=\left[\begin{array}{c|c}
A & B \\
\hline C & D
\end{array}\right]\left[\begin{array}{c}
v \\
r \\
p \\
\phi \\
\hline \delta_{\mathrm{dstab}} \\
\delta_{\mathrm{rud}} \\
\delta_{\mathrm{dsp}}
\end{array}\right]
$$

The state-space $[A, B, C, D]$ matrices for the 10.5-deg angle of attack and an airspeed of $137 \mathrm{kn}$ are as follows (note that the aircraft was linearized about a flight-path angle $\gamma$ of $0 \mathrm{deg}$ ):

$$
\left[\begin{array}{l|l}
A & B \\
\hline C & D
\end{array}\right]=
$$

$\left[\begin{array}{cccc|ccc}-0.116 & -227.3 & 43.02 & 31.64 & 0.062 & 0.101 & 0.0000 \\ 0.003 & -0.26 & -0.14 & 0 & -0.005 & -0.011 & -0.0016 \\ -0.021 & 0.67 & -1.37 & 0 & -0.047 & 0.004 & -0.0193 \\ 0 & 0.19 & 1.00 & 0 & 0 & 0 & 0 \\ 0 & 1.02 & 0.00 & 0 & 0 & 0 & 0 \\ \hline 0.247 & 0 & 0 & 0 & 0 & 0 & 0 \\ -0.001 & -0.98 & 0.19 & 0.14 & 0.0003 & 0.0004 & 0 \\ 0 & 0 & 57.3 & 0 & 0 & 0 & 0 \\ 0 & 57.3 & 0 & 0 & 0 & 0 & 0 \\ 0 & 0 & 0 & 57.3 & 0 & 0 & 0 \\ -0.003 & -0.008 & 0.05 & 0 & 0.0029 & 0.002 & 0.0004\end{array}\right]$




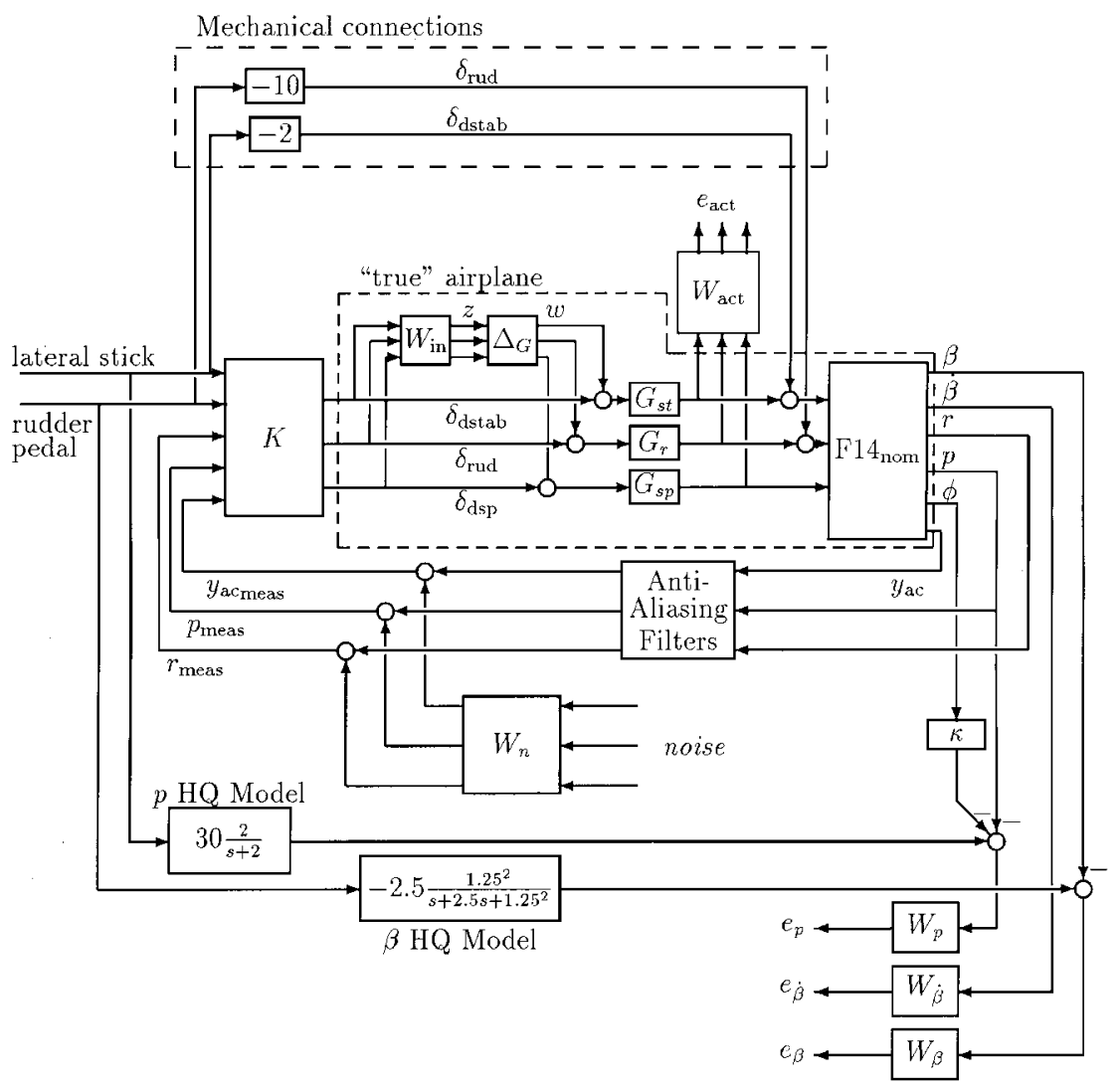

Fig. 2 F-14 control interconnection diagram with $\dot{\beta}$ weight.

The stabilizer actuators, $G_{\mathrm{st}}$ in Fig. 2, are modeled as a first-order system, $25 /(s+25)$. These actuators have \pm 12 -deg and $\pm 36-\mathrm{deg} / \mathrm{s}$ deflection and deflection rate limits. The series servos driving these actuators from the fly-by-wire control system have \pm 5 -deg and \pm 33 $\mathrm{deg} / \mathrm{s}$ deflection and deflection rate limits, respectively. The stabilizers operate in a differential manner.

The rudder actuators, $G_{r}$ in Fig. 2, are modeled as a first-ordersystem, $25 /(s+25)$. These actuators have \pm 30 -deg and \pm 125 -deg/s deflection and deflection rate limits. The series servos driving these actuators from the fly-by-wire control system have \pm 19 -deg and \pm 126 -deg/s deflection and deflection rate limits, respectively.

The F-14 aircraft has four spoiler panels per wing. They are commanded in pairs, resulting in four effective control surfaces. All four pairs can be deflected differentially for roll control and the inboard pairs deflected symmetrically for direct lift control. In this problem, the four spoiler control surfaces are ganged together for a single, differential spoiler command for roll. Each spoiler can be deflected through a range of $-4.5-55.0-\mathrm{deg}$. The total deflection limit is, therefore, $59.5 \mathrm{deg}$. Once on the glide path, it is assumed that the spoiler panels are trimmed at $+25 \mathrm{deg}$, allowing approximately \pm 30 deg of deflection. ${ }^{13}$ The spoiler actuators $G_{\text {sp }}$, driven directly by fly-by-wire control system, are modeled as a first-order system, $250 /(s+250)$ have a $\pm 250-\mathrm{deg} / \mathrm{s}$ deflection rate limit.

Roll rate, yaw rate, and lateral acceleration measurements are available for feedback. The roll-rate gyro is modeled as a secondorder system $157^{2} /\left(s^{2}+220 s+157^{2}\right)$ and measure rates up to $\pm 250 \mathrm{deg} / \mathrm{s}$. The yaw-rate gyro is modeled as a second-order system $137^{2} /\left(s^{2}+193 s+137^{2}\right)$ and measure rates up to $\pm 50 \mathrm{deg} / \mathrm{s}$. The lateral accelerometer is modeled as a second-order system $315^{2} /\left(s^{2}+440 s+315^{2}\right)$. This accelerometer can measure acceleration up to $\pm 1 \mathrm{~g}$.

The three measurement signals, roll rate, yaw rate and lateral acceleration, are passed through second-order, antialiasing filters prior to being fed to the controller. The natural frequency and damping values for the yaw-rate and lateral acceleration filters are $12.5 \mathrm{~Hz}$ and 0.5 , respectively and $4.1 \mathrm{~Hz}$ and 0.7 for the roll-rate filter. The antialiasing filters have unity gain at direct current (see Fig. 2).
The dashed box in Fig. 2 represents the true airplane, which corresponds to a set of F-14 plant models defined by $\mathcal{G}$. Inside the box is the nominal model of the airplane dynamics F14 nom; models of the actuators $G_{\mathrm{st}}, G_{r}$, and $G_{\mathrm{sp}}$; and two elements, $W_{\text {in }}$ and $\Delta_{G}$, which parametrize the uncertainty or errors in the model. This type of uncertainty is called multiplicative plant input uncertainty. The transfer function $W_{\text {in }}$ is assumed known and reflects the amount of uncertainty in the model. The transfer function $\Delta_{G}$ is assumed to be stable and unknown, except for the norm condition $\left\|\Delta_{G}\right\|_{\infty} \leq 1$.

In this example, we choose not to model the uncertainty in a detailed manner but rather to lump all types of model uncertainty together into three individual, complex, full-block multiplicative uncertainties at the input of the rigid-body aircraft nominal model. These account for errors in the differential stabilizer, rudder moment, and differentialspoiler coefficients. Modeling the uncertainty as three individual blocks is based on the assumption that isolated errors in the differential stabilizer, rudder moment, and differential spoiler channels do not couple to other channels. That is, an error in the differential stabilizer channel does not directly result in errors or uncertainties in the rudder and differential spoiler channels.

The differential stabilizers and rudder moment coefficients are linear with respect to deflections in the nonlinear simulation models. Unfortunately, the differential spoilers are highly nonlinear with respect to spoiler deflection. The roll spoiler moment coefficient varies from -0.0193 at the spoiler trim value to a peak of -0.095 at 14-deg deflection from trim of the spoilers. In the AFCS and DFCS, the spoiler actuators are not used in the feedback loop. Instead, a feedforward path from lateral stick to spoiler deflection is included to achieve the desired roll rates of the F-14 aircraft. The feedforward path basically inverts the nonlinear relationship between spoiler deflection and roll moment.

In the $\mu$ control designs, the differential spoilers are used in the feedbackloop. To average out the effect of the spoiler nonlinearities, the differential spoiler roll input coefficient of the nominal linear model is modified to represent the average moment generated by the spoilers when they are deflected. The nominal $C_{\delta_{\mathrm{sp}} p}$ value, $C_{\delta_{\mathrm{sp}} p_{\text {nom }}}=$ -0.0193 , which represents the $B(3,3)$ matrix coefficient, is scaled by 4.5 for the Kmod2q and Kmod2sx control design. 
The $\mu$ controllers make use of all three actuators in the feedback path. The differential spoiler actuators are treated as linear with multiplicative uncertainty in the problem formulation. This assumption makes the lateral-directional control problem difficult because the differential spoilers are the main actuators used to generate large roll rates on the F-14 aircraft. The multiplicative uncertainty weight is $W_{\text {in }}$ and is of the form $W_{\text {in }}(s):=\operatorname{diag}\left[w_{1}, w_{2}(s), w_{3}(s)\right]$. The $w_{1}$ weight associated with the differential stabilizer input is selected to be 0.038 . This represents a $3.8 \%$ error across frequency in the actuator model. The $w_{2}(s)=3(s+0.25) /(s+25)$ weight is associated with the rudder input and the $w_{3}(s)=3(s+5) /(s+200)$ weight is associated with the differential spoiler inputs. The values of $w_{1}$ and $w_{2}$ were interactively selected to provide some robustness below $1 \mathrm{rad} / \mathrm{s}$ and to limit the bandwidth of the designs. The value of $w_{1}$ was varied between 0.01 and 0.05 in the design process, with a final design value of 0.038 .

The stabilizer and rudder actuators are assumed to be more accurate than the spoiler actuator models. We know, based on the nonlinear F-14 models, that the aerodynamics associated with the spoilers have on the order of $\pm 50 \%$ uncertainty. In the design process, the uncertainty associated with the spoiler actuators is modeled to be about $7.5 \%$ at low frequency and increased to $100 \%$ uncertainty at $70 \mathrm{rad} / \mathrm{s}$ to achieve the desired roll-rate tracking objectives. Note that the weighting functions are used to normalize the size of the unknown perturbation $\Delta_{G}$. At any frequency $\omega$, the value of $\left|w_{1}(j \omega)\right|$ and $\left|w_{2}(j \omega)\right|$ can be interpreted as the percentage of uncertainty in the model at that frequency. The dependence on frequency of the uncertainty weight indicates that the level of uncertainty in the behavior of the aircraft depends on frequency.

The control design objective is to design a stabilizing controller $K$ such that for all stable perturbations $\Delta_{G}(s)$, with $\left\|\Delta_{G}\right\|_{\infty} \leq 1$, the perturbed closed-loop system remains stable, and the perturbed weighted performancetransferfunctions has an $\mathcal{H}_{\infty}$ norm less than 1 for all such perturbations. These mathematical objectives fit exactly in the structured singular-value $\mu$ framework.

The actuator deflections and deflection rates are weighted to ensure that they do not exceed their physical capabilities. Sensor noise is included in the problem formulation to mimic the real system. A diagram of the system interconnection structure used for control design and analysis is shown in Fig. 2. The diagram includes the feedback structure of the plant and controller and mechanical connections between the lateral stick and rudder pedal to differential stabilizers and the rudder, elements associated with the uncertainty models and performance objectives.

\section{B. Performance Objectives}

The performanceobjectiveis to have the true airplane, represented by the dashed box in Fig. 2, respond effectively to the pilot's lateral stick and rudder pedal inputs. The control problem is formulated as a model-matching problem in the $\mathcal{H}_{\infty}$ framework. The augmented F-14 aircraft is designed to track desired level-1 handling qualities models from the lateral stick to roll rate and from the rudder pedal to sideslip. ${ }^{14}$ The performance objectives include the following.

1) Response of the lateral stick, $\delta_{1 \mathrm{stk}}$, to roll rate $p$ and rudder pedals, $\delta_{\text {rudp }}$, to sideslip angle $\beta$ is decoupled. The lateral stick and rudder pedals have a maximum deflection of \pm 3.5 and \pm 3 in., respectively.

2) The aircraft handling quality (HQ) response from the lateral stick to roll rate should correspond to a first-order system

$$
30 \frac{2}{s+2} \frac{\mathrm{deg} / \mathrm{s}}{\text { in. }}
$$

A gain of 30 implies that a 1-in. deflection of the lateral stick will correspond to the aircraft rolling at a rate of $30 \mathrm{deg} / \mathrm{s}$. The aircraft HQ response from the rudder pedals to sideslip angle is

$$
-2.5 \frac{1.25^{2}}{s^{2}+2.5 s+1.25^{2}} \frac{\mathrm{deg}}{\text { in. }}
$$

The gain of 2.5 implies that a 1-in. differential deflection of the rudder pedals by the pilot will correspond to the aircraft yawing $2.5 \mathrm{deg}$. The minus sign in the HQ model is due to the sign convention of Grumman Corporation engineers. The HQ models are selected to achieve level-1 performance during the powered approach landing task.

\section{Performance Weights}

The results of the initial linear $\mu$ controllers implemented on the full-order, F-14 Fortran simulation indicated that it is important to penalize sideslip rate in addition to sideslip tracking of the rudder pedal commands. Therefore, we will concentrate on the design of controllers for $\alpha=10.5 \mathrm{deg}$ at an airspeed of $137 \mathrm{kn}$ with a penalty on $\dot{\beta}$. Including the $\dot{\beta}$ error term in the control problem is similar to the addition of a yaw damper to increase the damping of the Dutch roll mode (involving yaw and roll angle oscillations) of the aircraft. Classical designs make use of only the rudder to implement the yaw damper, whereas the multivariable $\mu$ designs make use of all three actuator surfaces to increase damping in the Dutch roll mode. ${ }^{10}$

The control objective is to achieve the desired closed-loop HQ characteristics that satisfy the pilot based on pilot-in-the-loopsimulations. As in the preceding section, it is assumed that the controllers measure or sense the lateral stick input, rudder pedal input, roll rate, yaw rate, and lateral acceleration. The controllers generate commands to the stabilizers, rudder, and spoilers.

\section{A. Kmod2q Weights}

Limits on the actuator deflection magnitude and rates are included in the control design via the $W_{\text {act }}$ weight. $W_{\text {act }}$ is a $6 \times 6$ constant, diagonal scaling matrix described by

$$
W_{\text {act }}=\operatorname{diag}\left(\frac{1}{83}, \frac{1}{16.7}, \frac{1}{315}, \frac{1}{25}, \frac{1}{480}, \frac{1}{268}\right)
$$

These weights correspond to relaxed weights on the stabilizer, rudder and spoiler deflection rate, and deflection limits for a \pm 1 -in. input. In this case, the deflection rate and deflection of the stabilizer actuators are penalized by $1 / 83$ and $1 / 16.7$, respectively. The lateral stick can deflect \pm 3 in., and the fly-by-wire control system is allowed to deflect the stabilizers $\pm 5 \mathrm{deg}$. Therefore, a \pm 1 -in. input should generate a maximum of $\pm 0.8 \mathrm{deg}$ of stabilizer deflection.

The weight $W_{n}$ corresponds to the sensor noise levels in the roll-rate $w n_{p}$, yaw-rate $w n_{r}$, and lateral acceleration, $w n_{\text {ac } y}$ channels. The sensor noise models are selected to represent the actual noise levels associated with each sensor. $W_{n}$ is a $3 \times 3$ diagonal, frequency-varying weight, $W_{n}:=\operatorname{diag}\left[w n_{p}, w n_{r}, w n_{\text {acy }}\right]$. The rate sensors are the same for roll and yaw, with a corresponding 0.1$\mathrm{deg} / \mathrm{s}$ noise level. The noise on the lateral accelerometer is modeled as $0.3(s+0.25) /(s+25)$, corresponding to increased noise in the acceleration measurement as a function of frequency.

The desired $\delta_{\text {stk }}$-to- $p$ and $\delta_{\text {rudp }}$-to- $\beta$ responses of the aircraft are formulated as a model-matching problem in the $\mu$ framework. The difference between the ideal responses, i.e., $\delta_{\text {lstk }}$ filtered through the roll-rate HQ model and $\delta_{\text {rudp }}$ filtered through the sideslip angle HQ model, and the aircraft response, $p$ and $\beta$, is used to generate an error that is to be minimized. The $W_{p}$ transfer function (see Fig. 2), weights the difference between the idealized roll-rate response and the actual aircraft response $p$. Here $\kappa$ is the amount of bank angle blended with roll rate to generate the aircraft desired roll-rate response; $\kappa$ is set to 0 in the $\mathrm{Kmod} 2 \mathrm{q}$ design. For the Kmod2q design

$$
W_{p}=0.038 \frac{0.145 s^{3}+59.47 s^{2}+1.47 s+0.012}{s^{3}+2.576 s^{2}+0.517 s+0.086}
$$

Because of a right-half-planezero at $0.05 \mathrm{rad} / \mathrm{s}$ in the transfer functions between $\delta_{\mathrm{dstab}}, \delta_{\mathrm{rud}}, \delta_{\mathrm{dsp}}$, and roll rate $p, W_{p}$ rolls off at low frequency to avoid requiring the lateral stick-to-roll rate transfer function to track sinusoidal signals below $0.05 \mathrm{rad} / \mathrm{s}$. The magnitude of $W_{p}$ between 0.2 and $2 \mathrm{rad} / \mathrm{s}$ is approximately 1 , corresponding to allowable roll-rate tracking error of $1 \mathrm{deg} / \mathrm{s}$. The performance weight on the $\beta$ tracking error, $W_{\beta}$, is $0.02(s+10) /(s+0.25)$. The $W_{\beta}$ weight allows for sideslip tracking errors on the order of $1.25 \mathrm{deg}$ below $0.2 \mathrm{rad} / \mathrm{s}$, which can increase to $50 \mathrm{deg}$ at $100 \mathrm{rad} / \mathrm{s}$. Plots of these weights, where $W_{p}$ is the solid line and $W_{\beta}$ is the dashed line, are shown in Fig. 3. 


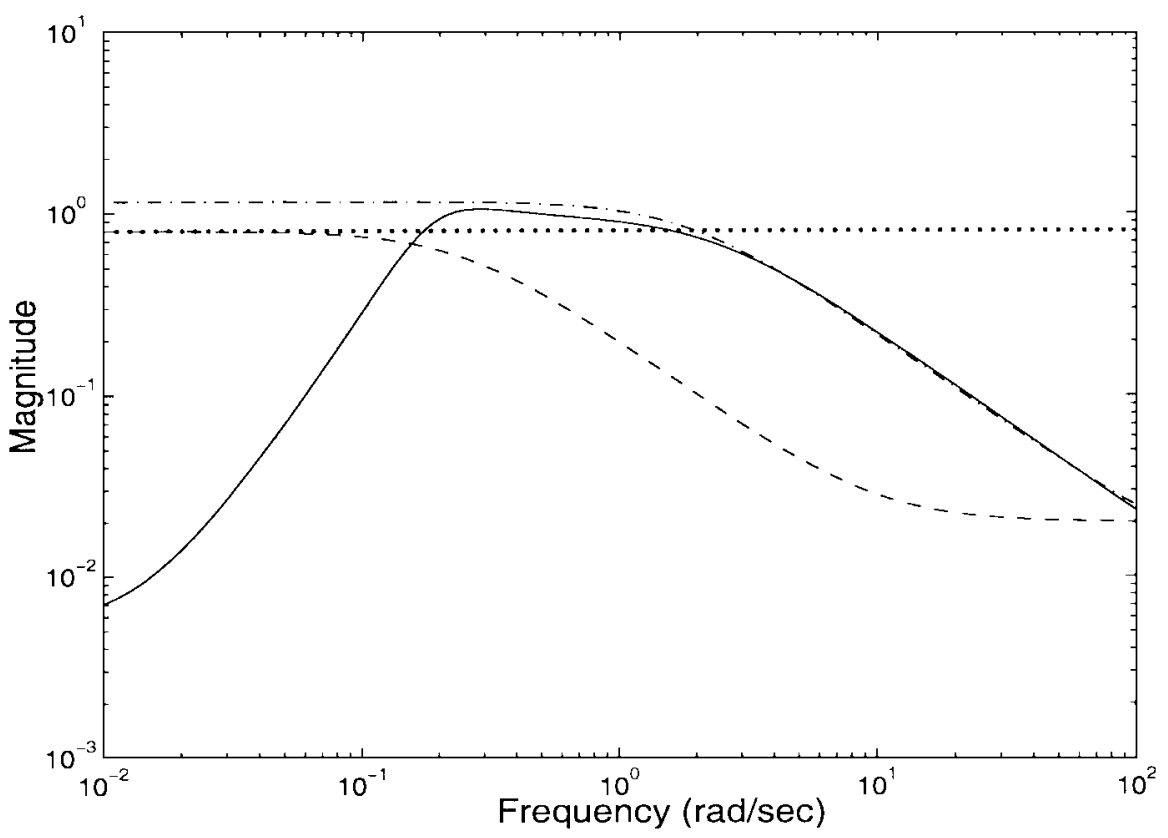

Fig. $3 W_{p}(\operatorname{Kmod} 2 q,-\longrightarrow), W_{\beta}(\operatorname{Kmod} 2 q,---), W_{p}(\operatorname{Kmod} 2 s x,-\cdot-)$, and $W_{\beta}(\operatorname{Kmod} 2 s x, \cdots \cdots)$ weighting functions.

$W_{\dot{\beta}}$ is a constant weight used to penalize the $\dot{\beta}$ output signal. This weight is used to characterize the pilot's desired directional response rate of the F-14 aircraft. Effectively, this weight is used to limit the bandwidth of the $\delta_{\text {rudp }}$-to- $\beta$ tracking channel and increase damping in the Dutch roll mode. In the Kmod2q control design, $W_{\dot{\beta}}$ weighting function is taken to be a constant 30 . The Kmod2q control design interconnection structure has a total of 19 states.

\section{B. Kmod2sx Weights}

There were two goals associated with the design of the Kmod2sx: 1) reduce the number of states in the control design model as compared with Kmod2q and 2) eliminate the effect of the right-halfplane zero associated with the lateral stick to roll-rate transfer function on the controller design. The second objective is handled by adding a small amount of the bank angle $\phi$ to the roll-rate response $p$. This results in the lateral stick to blended roll rate and bank angle being minimum phase while retaining the general transfer function response of the lateral stick to roll rate between 0.1 and $20 \mathrm{rad} / \mathrm{s}$. The objective of reducing the number of states was achieved in the following manner.

1) The first-order actuator models were replaced with constants (three state reduction).

2) The roll-rate HQ weight $W_{p}$ was reduced from a third-order weight to a first-order weight (two state reduction).

3) The $\beta$ HQ weight $W_{\beta}$ was reduced from a first-order weight to a constant (one state reduction).

4) The acceleration sensor noise model was reduced from a firstorder weight to a constant (one state reduction).

The total state order of the Kmod2sx control design interconnection structure was reduced from 19 states for $\operatorname{Kmod} 2 q$ to 12 states. The performance objectives for the Kmod2sx design were to achieve exactly the same time response characteristics as the Kmod2q design. A block diagram of the interconnection structure used for the design of Kmod2sx is shown in Fig. 2. Note that $G_{\mathrm{st}}, G_{\mathrm{sp}}$, and $G_{r}$ are all 1 in this diagram. Therefore, no weights on the deflection rates of the actuators are included in the control interconnection structure.

Limits on the actuator deflection magnitude are included via the $W_{\text {act }}$ weight. Note that because the actuator models were eliminated there are no rate measurement available. $W_{\text {act }}$ for the design of Kmod2sx was a $3 \times 3$ constant, diagonal scaling matrix described by $W_{\text {act }}=\operatorname{diag}(1 / 14,1 / 225,1 / 268)$. These weights correspond to the stabilizer, rudder, and spoiler deflection limits for a normalized \pm 1 -in. input from the lateral stick and rudder pedals. The input uncertainty weights in design Kmod2sx were the same as Kmod2q:
0.0375 for the differential stabilizer channel, $3(s+0.25) /(s+25)$ for the rudder channel, and $3(s+5) /(s+200)$ for the differential spoiler channel.

The desired $\delta_{\text {lstk }}$-to- $p$ and $\delta_{\text {rudp }}$-to- $\beta$ responses of the aircraft are formulated as a model-matching problem in the $\mu$ framework. The difference between the ideal responses, $\delta_{\text {lstk }}$ filtered through the rollrate HQ model, and $\delta_{\text {rudp }}$ filtered through the sideslip angle HQ model, and the aircraft response, a blended $p$ and $\phi$ is used to generate an error that is to be minimized. The blending variable $\kappa$ is set to 0.05 . The transfer function that weights the difference between the ideal roll-rate response and the actual aircraft roll-rate response is $W_{p}=0.012[(s+180.5) /(s+1.875)]$. The performance weight on the $\beta$ tracking error, $W_{\beta}$, is a constant 0.8 . $W_{p}$ allows for roll-rate tracking errors of $1 \mathrm{deg} / \mathrm{s}$ and sideslip tracking errors of $1.25 \mathrm{deg}$. Plots of these weights are shown in Fig. 3.

$W_{\dot{\beta}}$ weighting functions is taken to be a constant 30 , the same as in the $\mathrm{Kmod} 2 \mathrm{q}$ control design. As in $\mathrm{Kmod} 2 \mathrm{q}$ design, we desire a decoupled response between the roll and yaw axis. The differential spoiler $B$ matrix coefficient associated with the roll state was scaled by 4.5 in each design model to represent the spoiler nonlinearities.

\section{Controller Designs}

The control design block diagram shown in Fig. 2 results in an open-loop interconnection structure, $P(s)$, with 11 inputs and 17 outputs. The controllermeasures the lateral stick input, rudder pedal input, roll rate, yaw rate, and lateral acceleration. The controllers return commands to the differential stabilizers (degrees), rudder (degrees), and differential spoilers (degrees).

The continuous-time controllers are designed using the $\mu$ synthesis $D-K$ iteration control design procedure. The resulting controllers are discretized at a $60-\mathrm{Hz}$ sample rate, when implemented in the simulations. Initially, an $\mathcal{H}_{\infty}$ (sub)optimal controller for the open-loop interconnection $P$ is designed. This amounts to holding the scaling variables fixed at unity and minimizing the $\|\cdot\|_{\infty}$ norm of the closed-loop system over the controller variable $K$. This controller is denoted as $K_{1}$.

The second step in $D-K$ iteration involves a $\mu$ analysis corresponding to the closed-loop system. This calculation produces frequency-dependert scaling variables. In a general problem (with more than two blocks), there are several scaling variables, and the overall matrix is referred to as the $D$ scales. The varying variables in the $D$ scales are fit (in magnitude) with proper, stable, minimumphase rational functions and absorbed into the generalized plant for additional iterations. These scalings are used to trick the $\mathcal{H}_{\infty}$ minimization to concentrate more on minimizing $\mu$ rather than $\bar{\sigma}$ across frequency. 
The new generalized plant used in the second iteration has extra states, in addition to the original states of the generalizedplant $P(s)$. These extra states are due the $D$-scale data being fit with a rational function and are absorbed into the generalized plant for the next iteration. For each control design 2-4 $D-K$ iterations are performed until the $\mu$ reaches an equilibriumpoint, which usually was a $\mu$ value of approximately 1 . The reader is referred to Refs. 3-6, 8, and 9 for more information on $\mu$ synthesis and the $D-K$ iteration procedure.

Only the antialiasing filter associated with the roll-rate gyro is included in the control design model. The yaw rate and lateral acceleration antialiasing filters, as well as the series servos, are modeled as unity. This is done to limit the number of states of the controller and does not effect the controller design. All of the filters, actuator, and servo models are included in the nonlinear simulations.

\section{A. $K \operatorname{Kmod} 2 q$}

Kmod2q was designed via four $D-K$ iterations and resulted in a 33-state controller. This controller was reduced from 33 states to 20 states using balanced realization model reduction with little effect on the robust performance $\mu$ value. The 20 -statereduced-order controller, denoted as Kmod2q from now on, was implemented in the SIMULINK and Fortran F-14 aircraft nonlinear simulations.

The gain and phase margin of each loop is determined by individually breaking one feedback loop. The closed-loop system includes the series servo, the actuator models, nominal F-14 aircraft model, the sensor models, and the antialiasing filters. Table 1 contains the gain and phase margins and the frequency at which they are calculated of the input and output loops for $\operatorname{Kmod} 2 q$. The objective is to have $6 \mathrm{~dB}$ of gain margin and $45 \mathrm{deg}$ of phase margin in each input and output loop. Note that the differential spoiler $B$ matrix coefficient associated with the roll state was scaled by 4.5 in each gain and phase margin model to account for the spoiler nonlinearities. This scaling may be overly conservative for determining the gain and phase margins. Table 1 also contains the gain and phase margins and the frequency at which they are calculated of the input and

Table 1 Gain and phase margins for Kmod2q with scaled spoiler roll coefficient and nominal coefficient in parenthesis

\begin{tabular}{lcccc}
\hline \hline Channel & $\begin{array}{c}\text { Gain margin, } \\
\mathrm{dB}\end{array}$ & $\begin{array}{c}\omega_{\mathrm{GM}}, \\
\mathrm{rad} / \mathrm{s}\end{array}$ & $\begin{array}{c}\text { Phase margin, } \\
\mathrm{deg}\end{array}$ & $\begin{array}{c}\omega_{\mathrm{PM}}, \\
\mathrm{rad} / \mathrm{s}\end{array}$ \\
\hline$p$ & $4.0(3.3)$ & $16.9(0.04)$ & $22.0(60.0)$ & $11.5(4.3)$ \\
$r$ & $19.5(18.1)$ & $17.1(15.5)$ & $108.7(82.9)$ & $1.7(0.3)$ \\
$\mathrm{ac}_{y}$ & $10.2(9.9)$ & $0.4(0.4)$ & $68.1(57.0)$ & $0.1(0.1)$ \\
$\delta_{\text {stab }}$ & $19.8(23.9)$ & $16.7(16.1)$ & $\infty(114.2)$ & $N a N(0.2)$ \\
$\delta_{\text {rud }}$ & $19.8(19.1)$ & $14.3(14.2)$ & $109.4(107.4)$ & $0.1(1.9)$ \\
$\delta_{\text {sp }}$ & $4.2(17.4)$ & $17.0(17.6)$ & $23.2(70.8)$ & $11.6(3.8)$ \\
\hline \hline
\end{tabular}

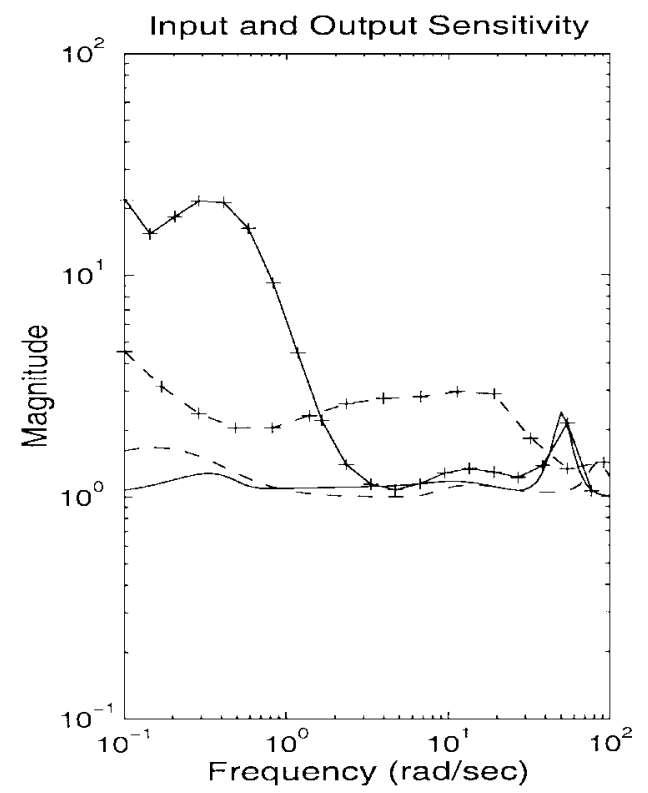

output loops for Kmod2q with the original differential spoiler $B$ matrix coefficient. These values are in parenthesis in the table. The roll-rate gain margin is at very low frequency and is not a problem.

Figure 4 contains plots of the input and output sensitivity and complementary sensitivity functions (Kmod2q is the solid line). The closed-loop models include the series servo, the actuator models, F-14 aircraft model, the sensor models, and the antialiasing filters. These plots provide insight into the robustness properties of the Kmod2q controller in the presence of modeling error at the input to the servoactuators and output of the F-14 linearized plant model. Between 0.1 and $10 \mathrm{rad} / \mathrm{s}$, the input sensitivity and complementary sensitivity functions have a maximum value of 2.2. This implies fully coupled, $45 \%$ input uncertainty would lead to instability. This indicates a very robust design. The output sensitivity and complementary sensitivity functions have values of approximately 20 between 0.1 and $1 \mathrm{rad} / \mathrm{s}$ and of 1 between 2 and $40 \mathrm{rad} / \mathrm{s}$. The value of 20 indicates that controller Kmod2q is sensitive to coupled uncertainty in the measurements in the frequency range of 0.1-1 rad/s. At crossover, the values of the output sensitivity and complementarysensitivity functions are about 1.3 indicating that the closed-loop system can tolerate up to $75 \%$ uncertainty in the sensor measurements at crossover. One reason for the higher sensitivity to uncertainty at outputs is due to the difference in measurement units. The roll rate is scaled to be 30 for a unit input, the yaw rate is scaled to be 5 , and the acceleration measurement will be approximately $0.1 \mathrm{~g}$. Therefore, the controller is sensitive to the large-gain coupling between the roll-rate measurement and acceleration measurement. In contrast, the input actuator command are all of the same magnitude.

The closed-loop response of the F-14 aircraft was simulated in both nonlinear simulations with the actautor and sensor models, antialiasing filters, lateral stick and rudder pedal inputs, and the discretized controllers sampled at $60 \mathrm{~Hz}$. The nonlinearities associated with the spoiler actuators are included in the SIMULINK simulation. The aerodynamic data are based on a single-point design and only the linearized lateral-directionalaxis dynamics of the F-14 aircraft are included in the SIMULINK model. The Fortran simulation contains the full six-degree-of-freedomEuler dynamics of the aircraft, full envelope aerodynamic data, and the nonlinearities associated with the actuator models. No noise was added to the sensor measurements in the simulations.

Consider the nonlinear simulation results of the Kmod2q controller. In each simulation, 1-in., 1-s doublet commands are commanded independentlyto the lateral stick and rudder.In Figs. 5 and 6 we show the $p, \phi, r, \beta$, and control surface deflection responses to a lateral stick doublet pair. From these we see that the roll response is very close to the HQ model. The roll-rate response of the aircraft to lateral stick is excellent, as seen in Fig. 5. All deflections are

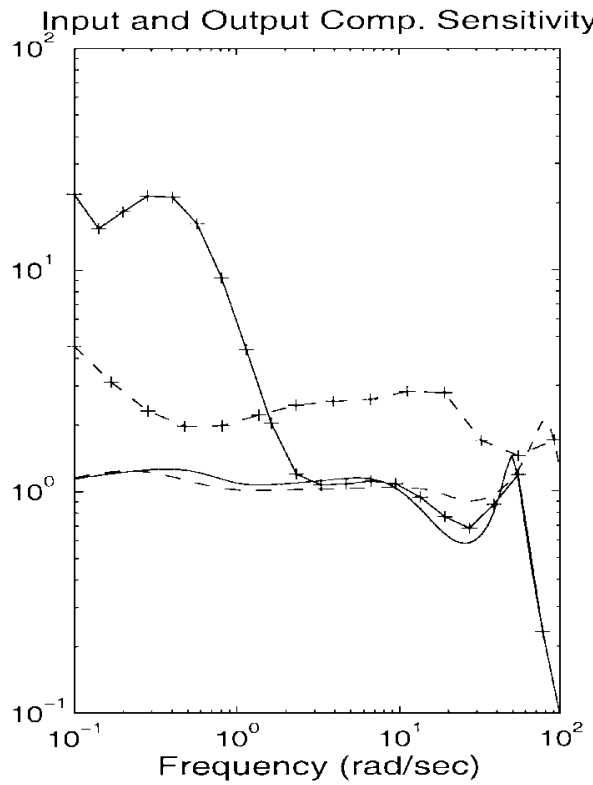

Fig. 4 Input and output (++) sensitivity and complementary sensitivity plots: $\operatorname{Kmod} 2 q(-)$ and $\mathrm{Kmod} 2 \mathrm{sx}(---)$. 

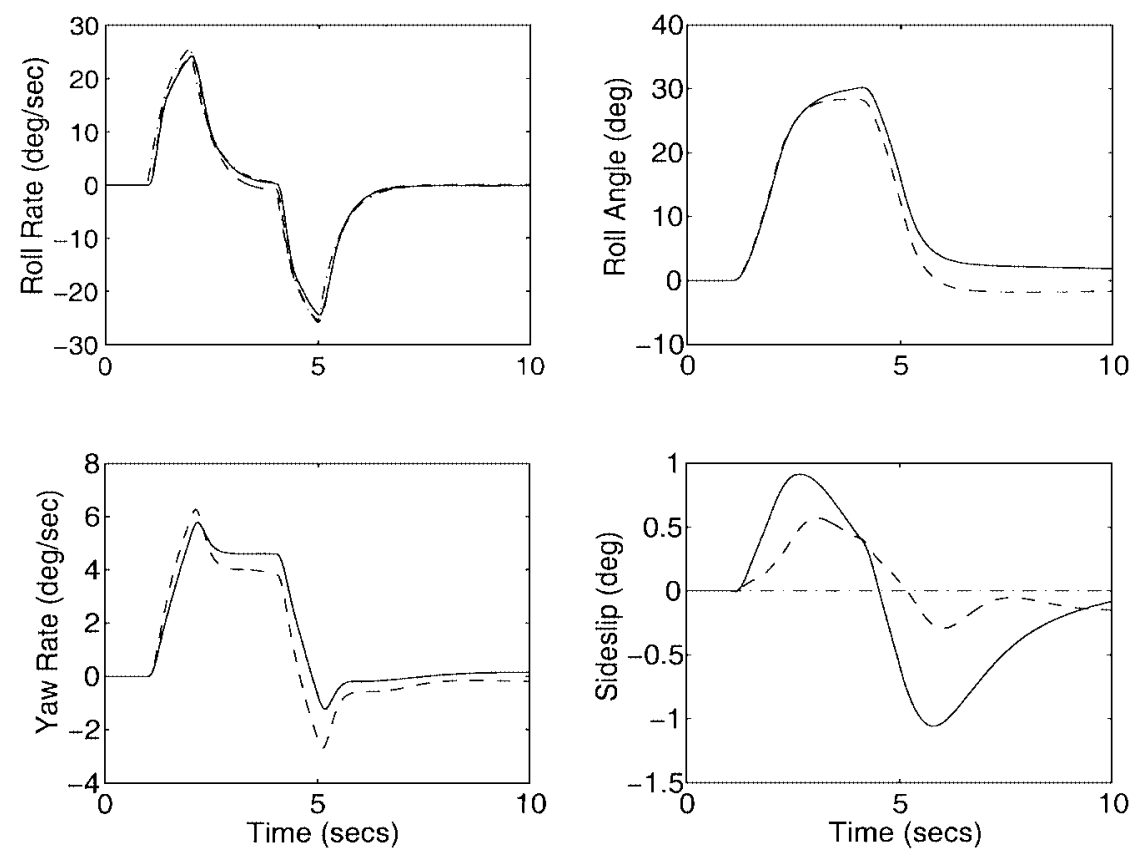

Fig. $5 \operatorname{Kmod} 2 q(-), \operatorname{Kmod} 2 s x(---)$, ideal response $(-\cdot) p, \phi, r$, and $\beta$ responses to a \pm 1.0 -in. lateral stick doublet input, $V_{\text {cal }}=137 \mathrm{kn}$ and $\alpha=10.5 \mathrm{deg}$.
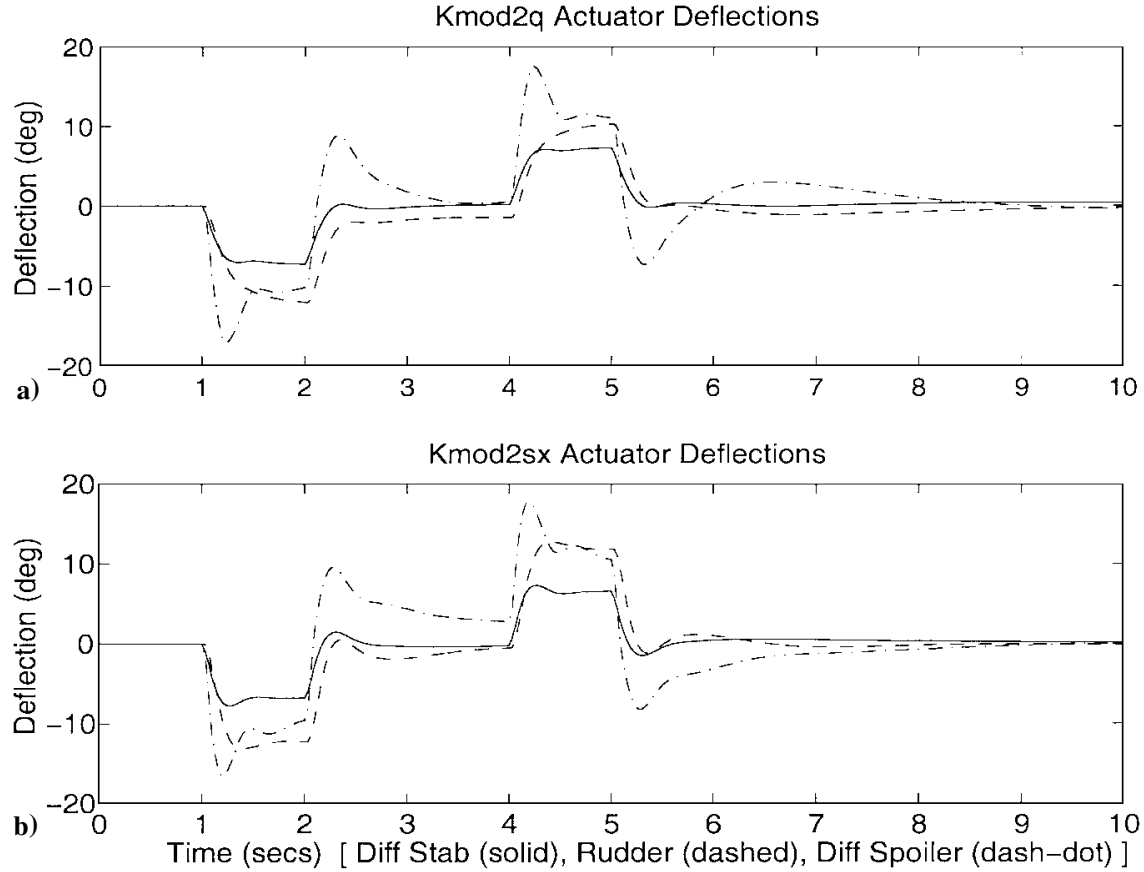

Fig. 6 Actuator commands to a \pm 1.0 -in. lateral stick doublet input, at $V_{\text {cal }}=137 \mathrm{kn}$ and $\alpha=10.5$ deg: a) $\mathrm{Kmod} 2 q$ and b) $\mathrm{Kmod} 2 \mathrm{sx}$.

well within their magnitude and rate limits. The $p, \phi, r, \beta$, and control surface deflection responses to a rudder pedal doublet pair are shown in Figs. 7 and 8. The sideslip angle $\beta$ is kept small during the lateral stick maneuver. The lateral response of $\operatorname{Kmod} 2 \mathrm{q}$ is agile due to a responsive rudder channel as seen in Fig. 7. The directional response of the aircraft is also very good.

There is excellent correlation between the nonlinear SIMULINK and Fortran simulations for all of the variablesexcept for the sideslip angle $\beta$. The sideslip angle is usually has a $30-40 \%$ larger response in the Fortran simulation compared with the SIMULINK response.

\section{B. Piloted Evaluation of Kmod2q}

On Feb. 6, 1996, Scott Kelly, lead F-14 test pilot for qualification of the DFCS, compared the AFCS, DFCS, and the $\mu$ controller design Kmod2q in the crewed flight simulator at U.S. Naval Air War- fare Center in Patuxent River, Maryland. The approach landing task was started at 1000 - $\mathrm{ft}$ altitude and the air speed was held between 128 and $142 \mathrm{kn}$. Controller Kmod2q designed for the 10.5-deg angle of attack linearized model were tested. The comments pertaining to the Kmod2q design are included. Note that the pronoun I in the following quotes corresponds to the comments of Kelly during the simulations.

Nominal and Off-Center Landings

I can make very precise bank angle corrections which is nice once you get use to it. I like the roll sensitivity of this design.

With controller Kmod2q, I had the nicest landing I've ever flown in the simulator.

(Controller Kmod2q) is less proverse than other (previous $\mu$ ) designs. The nose is basically neutral. I don't see anything wrong 

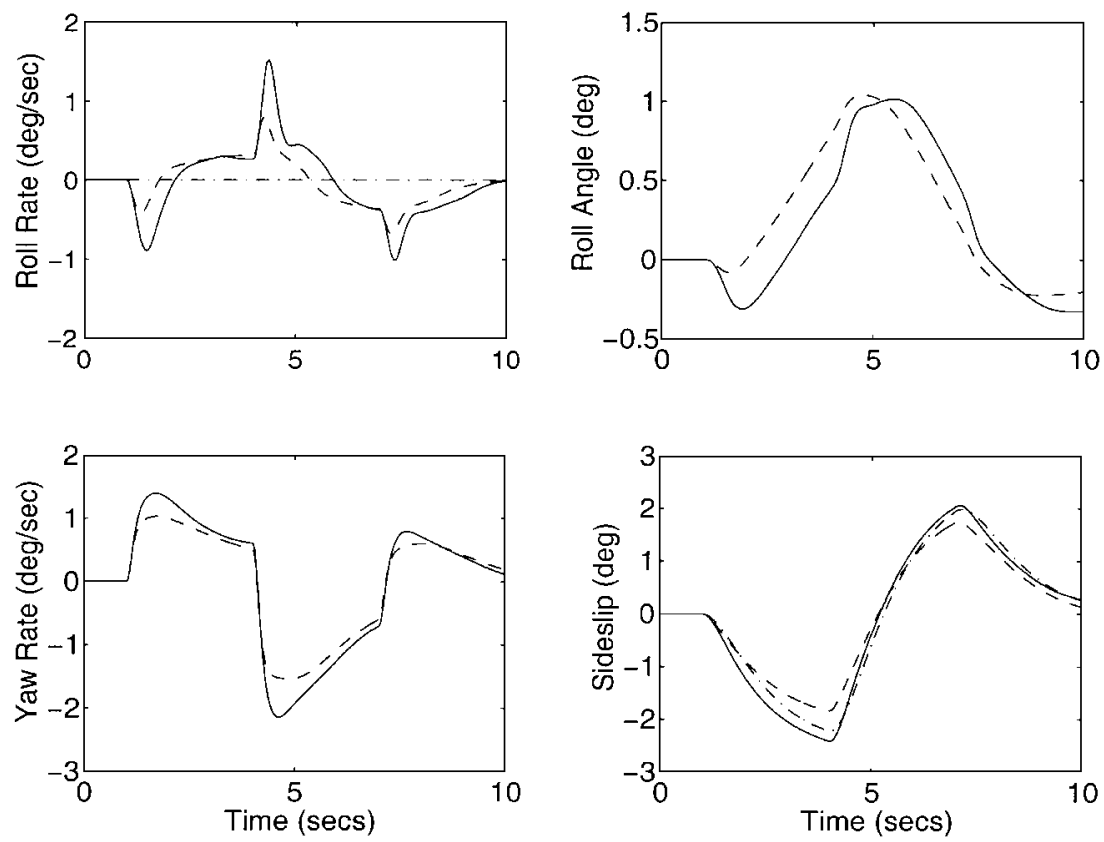

Fig. $7 \operatorname{Kmod} 2 q(-), \operatorname{Kmod} 2 s x(---)$, ideal response (- - - $p, \phi, r$, and $\beta$ responses to a \pm 1.0 -in. pedal input, $V_{\text {cal }}=137 \mathrm{kn}$ and $\alpha=10.5 \mathrm{deg}$.
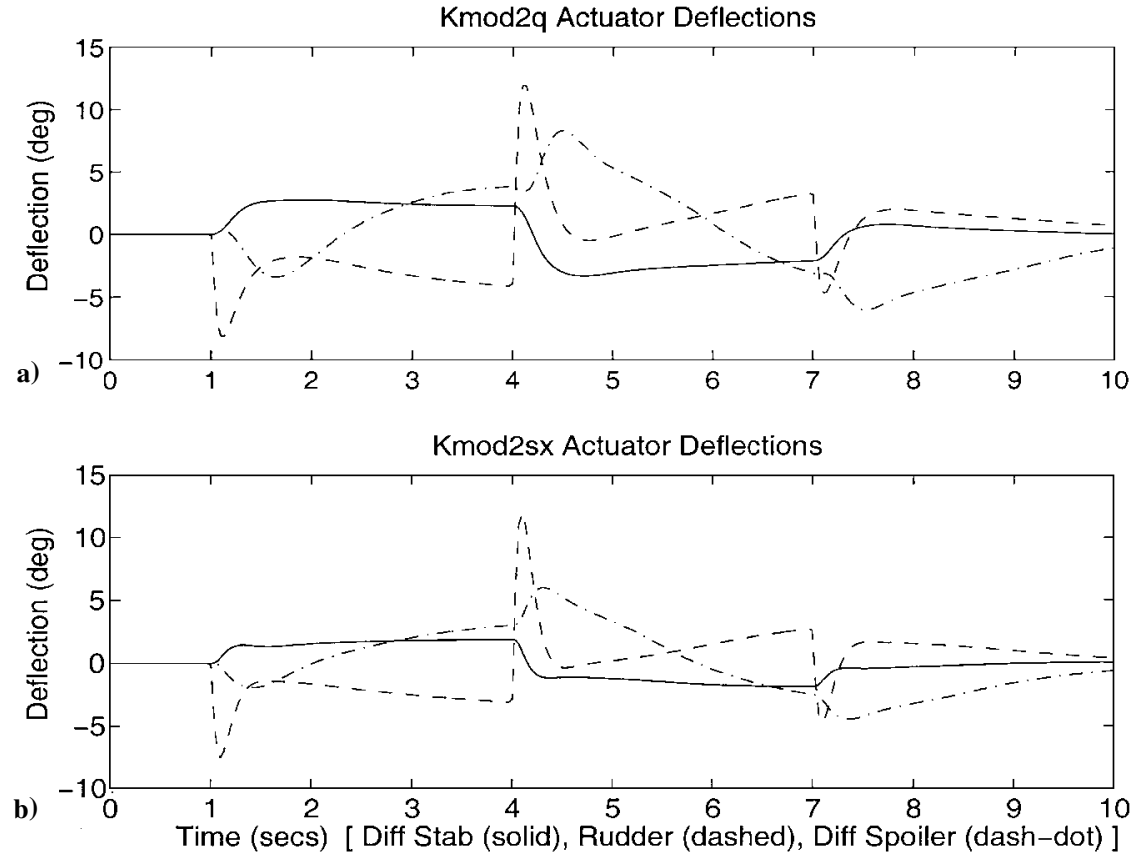

Fig. 8 Actuator commands to a \pm 1.0 -in. rudder pedal doublet input, at $V_{\text {cal }}=137 \mathrm{kn}$ and $\alpha=10.5$ deg: a) $\mathrm{Kmod} 2 q$ and b) $\mathrm{Kmod} 2 \mathrm{sx}$.

with this $(\operatorname{Kmod} 2 \mathrm{q})$ design. The nose is pointed where it is expected to be.

\section{Single-Engine Landings}

The aircraft exhibits little sideslip, less than 2 degrees. Its easy to land. In fact its easier to land this controller with a single engine that the AFCS with both engines.

There is nothing wrong with this $(\operatorname{Kmod} 2 \mathrm{q})$ design, which is rare for an F-14 aircraft. This is how I expect an airplane to fly. This controller $(\operatorname{Kmod} 2 \mathrm{q})$ is definitely nicer than the DFCS, no doubt about it.

10-Knot Cross Wind from Right with Two Engines

Pretty easy task to land aircraft (with the cross wind). I only have to touch the rudders at the end to land on the runway.
Aircraft is flying wings down on its own. The aircraft is putting in the lateral stick and rudder that's required.

Wrap Up Comments by Kelly

Overall I really like this (Kmod2q) controller: This is how I thought the airplane should fly. I thought it was the best flight control law I've flown in the F-14. The AFCS has a lot of deficiencies, the DFCS is a big improvement over that, but in the limited look I had in the simulator I really like what I saw of this design.

The bank and heading capture were really nice with small overshoots. I could control line up, which is very difficult in the AFCS F-14 normally, to within a couple of feet. The offset landing task was also easy to do.

Another thing I liked about it was that in single engine approach I didn't have to use the rudder. The (control) system fed in the 
Table 2 Gain and phase margins for $\operatorname{Kmod} 2 s x$

\begin{tabular}{lcccc}
\hline \hline Channel & $\begin{array}{c}\text { Gain margin, } \\
\mathrm{dB}\end{array}$ & $\begin{array}{c}\omega_{\mathrm{GM}}, \\
\mathrm{rad} / \mathrm{s}\end{array}$ & $\begin{array}{c}\text { Phase margin, } \\
\mathrm{deg}\end{array}$ & $\begin{array}{c}\omega_{\mathrm{PM}}, \\
\mathrm{rad} / \mathrm{s}\end{array}$ \\
\hline$p$ & 4.5 & 17.3 & 20.3 & 12.2 \\
$r$ & 13.8 & 13.7 & 103.2 & 2.2 \\
$\mathrm{ac}_{y}$ & 28.8 & 37.1 & $\infty$ & $\mathrm{NaN}$ \\
$\delta_{\text {stab }}$ & 14.7 & 15.1 & $\infty$ & $\mathrm{NaN}$ \\
$\delta_{\text {rud }}$ & 12.7 & 0.01 & 92.0 & 0.5 \\
$\delta_{\text {sp }}$ & 5.0 & 17.6 & 21.7 & 12.3 \\
\hline \hline
\end{tabular}

amount of rudder required to keep the sideslip between 0 and 2 degrees. This reduced sideslip due to the thrust asymmetry to a really small amount. This is a really important safety consideration when you are trying to land the aircraft aboard ship with the control laws you only have to fly with the stick and not the rudders. (This) means I can better control line up, glide slope, as well as pay more attention to landing the aircraft at a nominal approach speed which is really important. (This) is more important with (only a) single engine because if you are too slow the aircraft will depart.

\section{C. $K \bmod 2 s x$}

Kmod2sx was designed via two $D-K$ iterations. The resulting controllercontained 18 states. Balanced realization techniques were used to reduce the controller state order from 18 to 14 . Table 2 contains the gain and phase margins and the frequency at which they are calculated of the input and output loops for Kmod2sx. The differential spoiler $B$ matrix coefficient associated with the roll state was scaled by 4.5 in each gain and phase margin model to represent the spoiler nonlinearities, as discussed in Sec. IV.A. These achieve the desired gain and phase margins except in the roll rate and differential spoiler loops.

Figure 4 contains plots of the input and output sensitivity and complementary sensitivity functions with controller Kmod2sx (dashed lines). Between 0.1 and $10 \mathrm{rad} / \mathrm{s}$, the input sensitivity and complementary sensitivity functions have a maximum value of 2 , which implies a fully coupled, $50 \%$ input uncertainty would lead to instability. This is a very robust design. The output sensitivity and complementary sensitivity functions have a maximum value of 2.8 between 0.1 and $100 \mathrm{rad} / \mathrm{s}$, implying an uncertainty of size 0.35 would lead to instability. Kmod2sx has a factor of 7 higher level of robustness to modeling errors in the sensor measurements as compared with the $K \bmod 2 q$ design. The improved robustness of the $\operatorname{Kmod} 2 s x$ is due to the blending of $\phi$ and $p$, which eliminated the right-half-plane zero from the roll-rate tracking objective.

The closed-loop response of the F-14 aircraft with Kmod2sx was simulated using a simplified nonlinear model of the aircraft in SIMULINK and the full-order, Fortran nonlinear simulation of the F-14 aircraft. In each simulation, 1-in., 1-s doublet commands are commanded independently to the lateral stick and rudder. In Figs. 5 and 6 we show the $p, \phi, r, \beta$, and control surface deflection responses to a lateral stick doublet pair. From these we see that the roll response is very close to the HQ model. As in the Kmod2q design, the roll-rate response of the aircraft to lateral stick is excellent. The sideslip angle $\beta$ is kept small during the lateral stick maneuver, in fact smaller than the Kmod2q design. All deflections are well within their magnitude and rate limits. The $p, \phi, r, \beta$, and control surface deflection responses to a rudder pedal doublet pair are shown in Figs. 7 and 8. The lateral response of Kmod2sx to rudder pedal commands is not as responsive as the Kmod2q design, Fig. 7. The coupling of sideslip response to roll rate is reduced by a factor of 2 compared to the Kmod2q design.

\section{Pilot Comments During Simulation of Kmod2sx}

On Aug. 28, 1996, Alan Poindexter, the new lead test pilot for qualification of the DFCS, compared the AFCS, DFCS, and the linear on-speed ( $\alpha=10.5 \mathrm{deg}) \mu$ controller design $\mathrm{q}$ and $\mathrm{sx}$ in the crewed flight simulator at Patuxent River, Maryland. For the onspeed tests, the approach landing task was started at 1000-ft altitude and the air speed was held between 128 and $142 \mathrm{kn}$. Controllers Kmod2q and Kmod2sx designed for the 10.5-deg angle-of-attack linearized model were tested. The following comments pertain to the Kmod2sx design. Note that the pronoun I in the following quotes corresponds to the comments of Poindexter during the simulations.

Nominal and Off-Center Landings

$\mathrm{Kmod} 2 \mathrm{sx}$ has about the same responsiveness as Kmod2q, less sensitive in roll, no sideslip or proverse yaw. Not much difference between the two controllers.

Only a small amount of sideslip is generated (with Kmod2sx) with even aggressive $S$ turns a mile from touchdown, which are not standard inputs.

I really like it (Kmod2sx). It is not the same improvement as from the AFCS to the DFCS, but its close. This makes the flight simulator much easier to fly, and its usually much harder than the real aircraft.

I'd give the landing task with Kmod2q a Cooper-Harper (Ref. 15) rating of 3 (fair, some mildly unpleasant deficiencies) and with Kmod2sx a Cooper-Harper rating of 3, border-line 2 (good, negligible deficiencies). The deficiencies are due to the simulator and are related to the pitch-axis loop being sluggish (which was not part of this design). I'd give the lateral-directional handling qualities of Kmod2sx a Cooper-Harper rating of 2.

(Kmod2sx has) no degradation in glide slope control due to the use of the spoilers in the feedback path. Hard to believe this is an F-14. This is very nice.

\section{Summary and Conclusions}

A linear, multivariable controller for the F-14 lateral-directional axis during powered approach has been successfully designed and tested in pilot-in-the-loop simulations using structured singularvalue $\mu$ techniques. This work concentrated on achieving good HQ for pilot-in-the-loop simulations at the $\alpha=10.5 \mathrm{deg}$ and $V_{\text {cal }}=$ $137 \mathrm{kn}$ flight condition, nearly achieving the required gain and phase margin objectives. Based on the pilot comments, all of the control design objectives were achieved or exceeded. Controllers will also be designed for the $\alpha=2,6$, and 14 deg flight condition.

\section{Acknowledgments}

This work was performed by MUSYN Inc. under Contract N00421-94-R-0094 from the U.S. Naval Air Warfare Center at Patuxent River, Maryland. We would also like to thank Dale Enns for suggesting blending roll angle with roll rate to eliminate the effect of the low-frequency right-half-plane zero in the roll-rate response transfer functions.

\section{References}

${ }^{1}$ Durand, T. S., and Wasicko, R. J., "Factors Influencing Glide Path Control in Carrier Landing," Journal of Aircraft, Vol. 4, No. 2, 1965, pp. 146-158.

${ }^{2}$ Hughes, D., "Navy Begins Testing of F-14 Digital Controls," Aviation Week and Space Technology, July 24, 1995, p. 22.

${ }^{3}$ Stein, G., and Doyle, J. C., "Beyond Singular Values and Loop Shapes," Journal of Guidance, Control, and Dynamics, Vol. 14, No. 1, 1991,pp. 5-16.

${ }^{4}$ Balas, G. J., and Doyle, J. C., "Robustness and Performance Tradeoffs in Control Design for Flexible Structures," IEEE Transactions on Control Systems Technology, Vol. 2, No. 4, 1994, pp. 352-361.

${ }^{5}$ Balas, G. J., Doyle, J. C., Glover, K., Packard, A. K., and Smith, R., "The $\mu$ Analysis and Synthesis Toolbox,"MUSYN and MathWorks, Natick, MA, Nov. 1995.

${ }^{6}$ Balas, G. J., and Packard, A. K., "The Structured Singular Value $(\mu)$ Framework," CRC Controls Handbook, IEEE Press, Boca Raton, FL, 1996, Sec. 2.3.6.

${ }^{7}$ Doyle, J., Wall, J., and Stein, G., "Performance and Robustness Analysis for Structured Uncertainty," IEEE Conference on Decision and Control, Inst. of Electronical and Electronic Engineers, New York, 1982, pp. 629-636.

${ }^{8}$ Packard, A., Doyle, J., and Balas, G. J., "Linear, Multivariable Robust Control with a $\mu$ Perspective," Journal of Dynamic Systems, Measurement and Control, 50th Anniversary Issue, June 1993, pp. 426-438.

${ }^{9}$ Reiner, J., Balas, G. J., and Garrard, W., "Design of a Flight Control System for a Highly Maneuverable Aircraft Using Robust Dynamic Inversion," Journal of Guidance, Dynamics, and Control, Vol. 18, No. 1, 1995, pp. 18-24. 
${ }^{10}$ Blight, J. D., Dailey, L. R., and Gangsaas, D., "Practical Control Law Design for Aircraft Using Multivariable Techniques," International Journal of Control, Vol. 59, No. 1, 1994, pp. 93-137.

${ }^{11}$ Adams, R., Sparks, A., and Banda, S., "Full Envelope Multivariable Control Law Synthesis for a High Performance Test Aircraft," Journal of Guidance, Control, and Dynamics, Vol. 16, No. 5, 1993, pp. 948-955.

${ }^{12}$ Doyle, J., Glover, K., Khargonekar, P., and Francis, B., "State-Space Solutions to Standard $\mathcal{H}_{2}$ and $\mathcal{H}_{\infty}$ Control Problems," IEEE Transactions on Automatic Control, Vol. 34, No. 8, 1989, pp. 831-847.

${ }^{13}$ Niewoehner, R. J., and Kaminer, I. I., "Design of an AutoLand Con- troller for a Carrier-Based F-14 Aircraft Using $\mathcal{H}_{\infty}$ Output-Feedback Synthesis," Proceedings of the American Control Conference (Seattle, WA), Vol. 3, Inst. of Electrical and Electronics Engineers, Piscataway, NJ, 1994, pp. 2501-2505.

${ }^{14}$ Teper, G. L., and Stapleford, R. L., "An Assessment of the LateralDisectional Handling Qualities of a Large Aircraft in the Landing Approach," Journal of Aircraft, Vol. 3, No. 3, 1966, pp. 201-207.

${ }^{15}$ Cooper, G. E., and Harper, R. P., "The Use of Pilot Rating in the Evaluation of Aircraft Handling Qualities," NASA-TN-D-5153, April 1969. 